\title{
A quantum theory account of order effects and conjunction fallacies in political judgments
}

\author{
James M. Yearsley ${ }^{1,2}$ • Jennifer S. Trueblood ${ }^{1}$
}

Published online: 6 September 2017

(C) Psychonomic Society, Inc. 2017

\begin{abstract}
Are our everyday judgments about the world around us normative? Decades of research in the judgment and decision-making literature suggest the answer is no. If people's judgments do not follow normative rules, then what rules if any do they follow? Quantum probability theory is a promising new approach to modeling human behavior that is at odds with normative, classical rules. One key advantage of using quantum theory is that it explains multiple types of judgment errors using the same basic machinery, unifying what have previously been thought of as disparate phenomena. In this article, we test predictions from quantum theory related to the co-occurrence of two classic judgment phenomena, order effects and conjunction fallacies, using judgments about real-world events (related to the U.S. presidential primaries). We also show that our data obeys two a priori and parameter free constraints derived from quantum theory. Further, we examine two factors that moderate the effects, cognitive thinking style (as measured by the Cognitive Reflection Test) and political ideology.
\end{abstract}

Keywords Quantum probability theory · Order effects · Conjunction fallacy $\cdot$ Individual differences $\cdot$ Rationality

Electronic supplementary material The online version of this article (https://doi.org/10.3758/s13423-017-1371-z) contains supplementary material, which is available to authorized users.

James M. Yearsley

james.yearsley@gmail.com

1 Department of Psychology, Vanderbilt University, PMB 407817, 2301 Vanderbilt Place, Nashville, TN 37240-7817, USA

2 Department of Psychology, University of London, Northampton Square, London EC1V 0HB, UK
Every day we make hundreds of judgments about the world around us (e.g., How likely is it to rain tomorrow? How likely is it that Democrats will be the majority in the Senate after the next election?). A core question of human behavior is whether people's judgments and decisions can be considered normative. That is, do people behave as ideal decision makers, acting in a fully rational manner? Decades of research in human judgment and decision making suggests that the answer is no. People often make incorrect or biased decisions (Kahneman \& Tversky, 1972; Tversky \& Kahneman, 1975). If people's judgments violate normative rules, then what rules (if any) do they follow?

In recent years, an alternative class of decision-making models, based on the mathematics of quantum theory, have been developed to model situations where behavior is at odds with the normative predictions of classical probability theory (Busemeyer \& Bruza, 2012). Cognitive models based on quantum probability theory are computational-level models, focusing on the principles and representations guiding human behavior (cf. Griffiths, Chater, Kemp, Perfors, \& Tenenbaum, 2010). One key advantage of using quantum theory as a modeling framework is that it explains multiple types of judgment errors using the same basic machinery, unifying what have previously been thought of as disparate phenomena. The ultimate objective is to provide a computational-level framework for nonnormative decision making, allowing new predictions and a greater understanding of why failures of normative prescription arise, something not possible with models that treat different nonnormative behaviors in isolation.

In the present work, we examine two classic examples of nonnormative judgments: conjunction fallacies and order effects. The conjunction fallacy occurs when individuals judge the conjunction of two events to be greater than at least one of the constituents (Tversky \& Kahneman, 1983). For example, a conjunction fallacy occurs when the conjunction "tomorrow it will be sunny AND warm: is judged more likely than the 
single event "tomorrow it will be sunny." Order effects occur when the presentation order of evidence influences the perceived likelihood of a hypothesis. A typical finding is that given two pieces of evidence, $X$ and $Y$, where $X$ supports a hypothesis (e.g., starting a new exercise program) and $Y$ does not (e.g., maintaining a poor diet), the probability that individuals assign to the hypothesis $H$ (e.g., future weight loss) after hearing the evidence in the order $X, Y$ is less than the probability they assign when the evidence is presented in the order $Y, X$ (Hogarth \& Einhorn, 1992; Trueblood \& Busemeyer, 2011).

Quantum models account for these two effects through the incompatibility of events. This technical term from quantum probability theory means that events do not commute (i.e., $\operatorname{pr}(\mathrm{X} \& \mathrm{Y}) \neq \operatorname{pr}(\mathrm{Y} \& \mathrm{X}))$. Thus, by definition, incompatibility implies the presence of order effects (Trueblood \& Busemeyer, 2011; Wang \& Busemeyer, 2013). Incompatibility has also been used to explain conjunction fallacies (Busemeyer, Pothos, Franco, \& Trueblood, 2011). Since both order effects and conjunction fallacies arise from the same underlying assumption of incompatibility, one important prediction is that these effects should co-occur; a prediction we will test in the present study. In addition, quantum models predict quantitative constraints on the types of behavior we expect to see. Specifically, we examine two a priori and parameter-free constraints on the quantum theory predictions, one for order effects and one for conjunctions.

To test the quantum model predictions, we examine the normative status of real-world judgments about U.S. politics. In our experiment, participants reported their beliefs about the likelihood that five of the main candidates for the Republican and Democratic presidential nominations (Ted Cruz, Marco Rubio, Donald Trump, Hillary Clinton, and Bernie Sanders) would win various combinations of state primaries and the ultimate nomination. The overall aim is to understand whether there are nonnormative effects in these real-world judgments and whether they are consistent with the predictions of quantum theory. We also show that individual differences in the degree of nonnormative behaviors are associated with cognitive thinking style and political ideology.

\section{Method}

\section{Participants}

The experiment was conducted using the Qualtrics survey platform, with participants recruited via Amazon Mechanical Turk. The eligibility criterion was set to allow U.S. residents only, but no other restriction was placed on participation. Twelve hundred individuals (624 male) were recruited and paid $\$ 1.50$ each. As described below, each participant answered questions about one of two Democratic candidates (Hillary Clinton and Bernie Sanders) and one of three
Republican candidates (Ted Cruz, Marco Rubio, Donald Trump). The sample size was chosen so that there would be 400 participants for each Republican candidate.

\section{Materials and procedure}

The main judgments participants were asked to make concerned the likelihood that a given candidate would win various combinations of two chosen primaries and the ultimate party nomination. Questions took one of three forms: either a judgment about a single event, such as "How likely do you think it is that Ted Cruz will win the primary in Texas?"; a conditional, such as "Suppose Donald Trump wins the primary in Texas. How likely do you think it is that he will then win the nomination to be the Republican Presidential candidate?"; or a conjunction, such as "How likely do you think it is that Bernie Sanders will lose the primary in Massachusetts and lose the nomination to be the Democratic Presidential candidate?" For each question, participants responded on a 17-point Likert scale, with verbal labels adapted from Windschitl and Wells (1996). For a given candidate, there were four blocks of questions, each containing an order question (described in more detail below) and various other questions. Questions within a block were presented in a random order, and the order of blocks was randomized.

Some of the questions asked participants to make a sequence of decisions about the likelihood a candidate would win the nomination as they learned new information about their performance in the primaries. For example, they might be asked about the likelihood than Donald Trump would win the nomination $(N)$ given that he wins the primary in Texas, (e.g. $p(N \mid T X)$ ). Participants might then be asked to reevaluate Trump's chances given that he also loses the primary in Virginia (e.g., $p(N \mid T X, \overline{V A})$ ). Information about the outcome of the first primary remained on the computer screen when new information about the second primary was presented, so participants had access to this during their subsequent choice.

The primaries we selected occurred on the same day, the first Super Tuesday of the 2016 U.S. presidential primaries (March 1, 2016), ensuring there was no direct causal link between them (participants were made aware of this fact.) This is critically important for testing order effects. If the primaries occurred on different days, the results of one could potentially influence the outcome of the other. In this case, order effects might be rational. For the Democrats, the primaries chosen where Massachusetts (MA) and Oklahoma (OK). For the Republican candidates, the primaries chosen were Texas (TX) and Virginia (VA).

After the main part of the task, participants were asked an additional set of questions designed to measure individual differences that might relate to a participant's judgments. Participants first answered a version of the Cognitive 
Reflection Test (CRT; Frederick, 2005), in the revised form suggested by Finucane and Gullion (2010). The CRT is designed to discriminate between participants adopting either a more intuitive or a more deliberative thinking style (Toplak, West, \& Stanovich, 2011). Full details of the questions are given in the supplementary materials. We expect that this measure will predict the presence and size of deviations from normative reasoning.

Participants then answered a number of questions relating to their political beliefs (these questions were asked after the main part of the study in order to avoid biasing responses). Participants were asked whether they were affiliated with a political party, and a self-reported ideology question (options: extreme conservative, moderate conservative, moderate, moderate liberal, extreme liberal). Participants finally answered a 10-point Ideological Consistency Scale designed by the Pew Research Center (2014). This is a list of 10 pairs of statements, and participants must pick the statement from each pair that they most agree with. Each pair consists of a statement more typically associated with a liberal ideology, and one more typically associated with a conservative ideology. Full details of the questions are given in the supplementary materials.

\section{Results}

All subjects were included in the analyses. In the body of this article, we only report Bayesian statistical tests performed using JASP (JASP Team, 2016), classical versions of all tests can be found in the Supplementary Material. The data and the Qualtrics survey code used to collect it are available on the Open Science Framework (https://osf.io/ucs8z/).

\section{Order effects}

To examine the presence of order effects we performed Bayesian paired-samples $t$ tests to test the hypothesis that $p(N \mid A, B)$ differs from $p(N \mid B, A)$, where $A$ and $B$ are different possible primaries - for example, win Oklahoma $(O K)$ or lose Texas $(T X)$. There are two possible order effect comparisons per candidate for a total of 10 comparisons across all five candidates. The Bayes factors (BF) for all 10 comparisons were greater than $10^{14}$, indicating extreme evidence for order effects. ${ }^{1}$ Group average data are presented in Fig. 1, from which we see the general pattern that the perceived likelihood of a candidate winning the nomination $N$ given that they win primary $A$ and lose primary $B, p(N \mid l o s e B$, win $A)$, is rated as higher on average than $p(N \mid$ win $A$, lose $B)$. In the language of decision making, this is referred to as a recency effect

\footnotetext{
${ }^{1}$ The Bayes factor $\mathrm{BF}_{10}$ is the ratio of evidence for $H_{1}$ over $H_{0} . \mathrm{BF}_{10}>1$ denotes support for the alternative hypothesis and $\mathrm{BF}_{10}<1$ support for the null hypothesis.
}

(Hogarth \& Einhorn, 1992). These results were confirmed by a Bayesian repeated-measures ANOVA examining two factors: Presentation Order and Candidate Pair. The model Presentation Order $\times$ Candidate Pair was preferred to all other models $\left(\mathrm{BF}_{\mathrm{M}}>1,000\right.$, extreme evidence) and to the null model $\left(\mathrm{BF}_{10}>1,000\right.$, extreme evidence). The analysis of effects also showed that the Bayes factor for inclusion of the two variables Presentation Order and Candidate Pair was very large $\left(\mathrm{BF}_{\text {inclusion }}>1,000\right)$. The analysis of effects is shown in Table 1, and full details are given in the Supplementary Material. Note that the presence of order effects in the data means that it is consistent with the basic assumption of incompatibility in quantum probability theory.

\section{Conjunction fallacies}

Next, we examine the presence of conjunction fallacies. We performed a series of one-sided Bayesian paired-samples $t$ tests to test the hypothesis that $p(X \wedge Y)>\min (p(X), p(Y))$, where $X$ and $Y$ are different possible events such as winning the nomination and losing a particular primary.

For each candidate, we measured eight different conjunctions for a total of 40 comparisons across the five candidates. Thirty-seven out of the 40 comparisons had $\mathrm{BF}_{10}>12$, strong evidence for conjunction fallacies. Full details of these tests are given in the Supplementary material. We also plot $p(X \wedge Y)$ vs $\min (p(X), p(Y))$, averaged across participants in Fig $2 \mathrm{a}$. Since almost all points lie above the diagonal we see that almost all conjunctions give rise to a fallacy. In Fig $2 \mathrm{~b}$ we plot the same conjunctions against $\max (p(X), p(Y))$, which allows us to quickly see that there are no double conjunction fallacies - that is, $p(X \wedge Y) \leq \max (p(X), p(Y))$. This is discussed in more detail below.

\section{Testing the quantum constraints}

Quantum theory predicts the following constraint on order effects, which we derive in the Supplementary Material:

$p(N \mid \bar{B}, A)-p(N \mid A, \bar{B})=p(N \mid \bar{A}, B)-p(N \mid B, \bar{A})$,

where $N$ represents a candidate winning the nomination, and $A$ and $\bar{A}$ represent the candidate winning or losing the primary A, respectively. The constraint arises because, at least in the simplest quantum models, the conditional probability of $N$ given a sequence of prior events (e.g., $A$ then $B$ ) depends only on the relationship between $N$ and the final event (e.g., $B$ ) in the sequence (mathematically, this relationship is quantified by the angle between the two events). In particular, $p(N \mid \ldots$, $A)=\cos ^{2}\left(\theta_{N A}\right)$ and $p(N \mid \ldots, \bar{A})=\sin ^{2}\left(\theta_{N A}\right)$, from which Equation (1) follows. This constraint is very strong in that it is parameter free. 


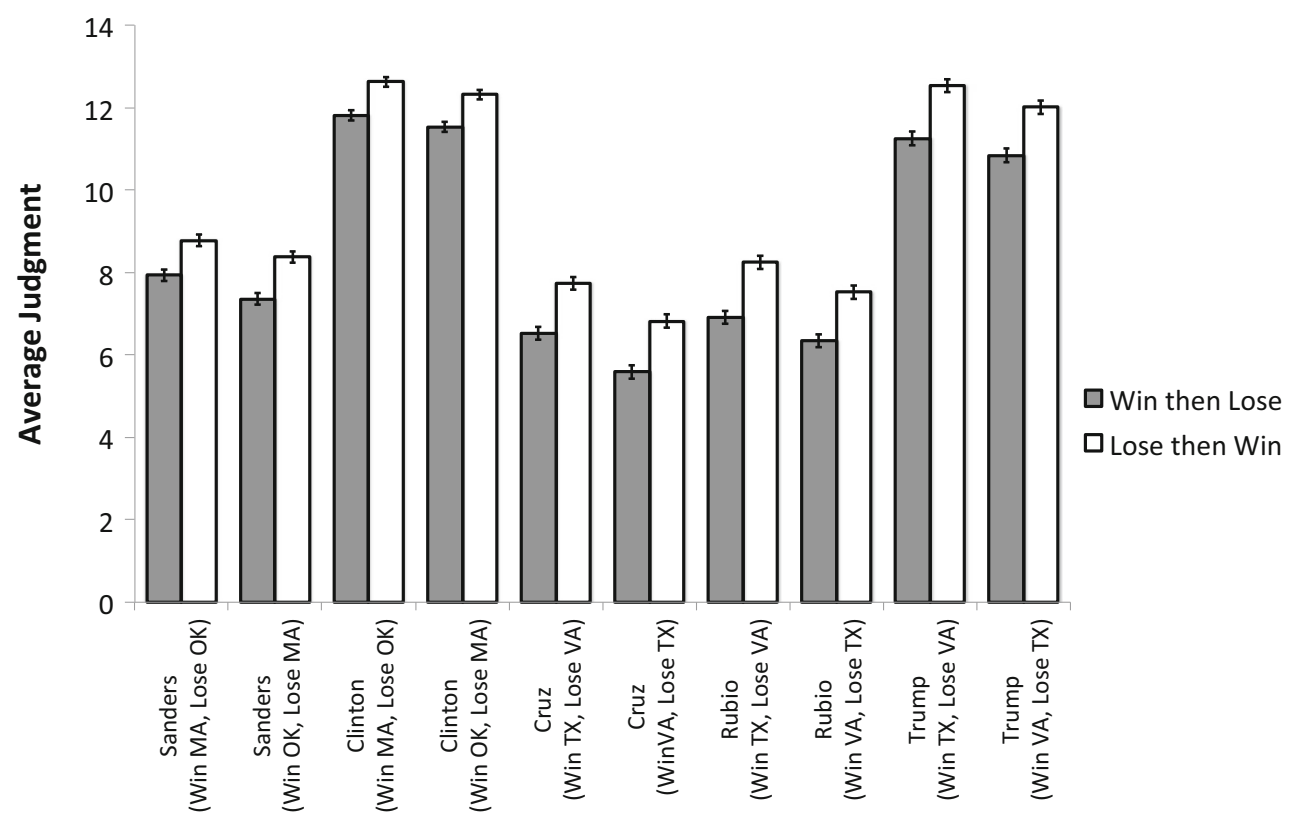

Fig. 1 Average judgment for each of the candidates and each of the possible win/lose combinations and orders. Conditionals involving first a loss and then a win are always given a higher likelihood, suggesting a recency effect. Error bars show $S E$ of the mean

This equality gives rise to five constraints, one for each candidate. We carried out a Bayesian repeated-measures ANOVA, with the left and right hand sides of Equation (1) as one factor, and candidate type as the other. The particular pair of candidates a participant saw was coded as a betweensubjects factor. If the quantum constraint, Equation (1), is obeyed, we expect to see no effect of Side, although possibly effects due to Candidate Type and Candidate Pair. A model with Candidate Type (Republican or Democrat) was preferred to all other models $\left(\mathrm{BF}_{\mathrm{M}}=391\right.$, extreme evidence $)$ and to the null model $\left(\mathrm{BF}_{10}=5.07 \times 10^{4}\right.$, extreme evidence). The analysis of effects is shown in Table 2, and full details are given in the Supplementary Material. It shows that only the effect Candidate Type has a BF $>1$, representing evidence for inclusion. Crucially, there was no support for the inclusion of the variable Side. The data is therefore consistent with this constraint arising from the quantum model.

Quantum theory may also be used to derive constraints on conjunctions. First, regardless of the particular model, quantum theory does not permit double conjunction fallacies. The reason for this is that while probabilities in quantum theory do not obey all the usual sum rules, they do obey some of them.

Table 1 Analysis of effects for the Bayesian repeated-measures ANOVA testing for order effects (candidate pair and presentation order are the repeated measures)

\begin{tabular}{ll}
\hline Effects & $B F_{\text {Inclusion }}$ \\
\hline Presentation Order & $\infty$ \\
Candidate Pair & $\infty$ \\
Presentation Order * Candidate Pair & $5.77 \times 10^{-5}$ \\
\hline
\end{tabular}

In particular, when the probability for a sequence of events is computed, the final event in the sequence will obey the usual sum rules. Therefore given two events, $X, Y$, the probability sum rules will always be satisfied for one of them.

We tested for the presence of double conjunction fallacies by performing a series of Bayesian paired-samples $t$ tests, similar to the tests for regular conjunction fallacies, but where the comparison is now between $p(X \wedge Y)$ and $\max (p(X), p(Y))$. The full results are given in the Supplementary Material, but all Bayes factors were less than 0.015 , indicating very strong evidence for the absence of double conjunction fallacies.

Secondly, quantum theory may be used to derive upper and lower bounds on the probability of the conjunction of two events, given their individual probabilities. Full details are given in the Supplementary Material, but the intuition is that the joint probability can be written in terms of the conditional $p(Y \mid X)$, which depends only on the angle between $X$ and $Y$. We can bound this angle if we know the angles between $X, Y$ and the initial state vector (the initial state vector represents an individual's beliefs before any questions are asked), and these can be determined from $p(X), p(Y)$. Some algebra shows,

$$
\begin{aligned}
& p_{\max / \min }(X \wedge Y) \\
& =p(X)\left[\begin{array}{l}
p(X) p(Y)+(1-p(X))(1-p(Y)) \\
\pm 2 \sqrt{p(X)(1-p(X)) p(Y)(1-p(Y))}
\end{array}\right]
\end{aligned}
$$

For example suppose $p(X)=.75$ and $p(Y)=.25$, then quantum theory requires $0.14 \leq p(X \wedge Y) \leq 0.42$. 


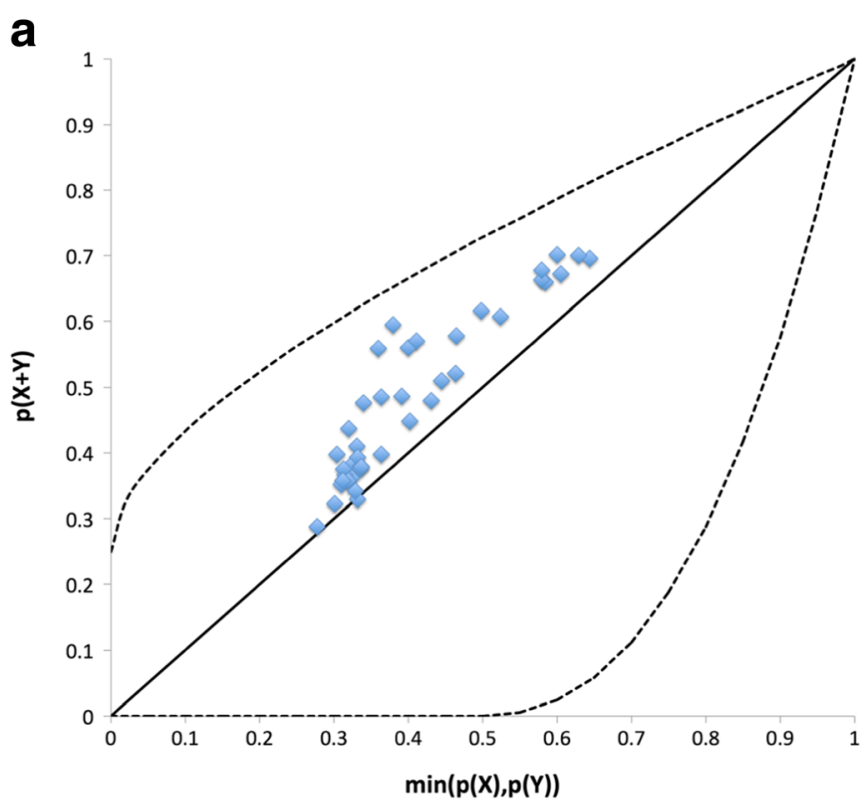

Fig. 2 Mean conjunction judgments plotted against the minimum or maximum conjunct. a Conjunctions plotted against the minimum conjunct, almost all points lie above the diagonal, indicating a conjunction fallacy. The dotted lines represent the quantum bounds. b

In Fig. 2a-b we plot the maximum and minimum allowed values of the conjunction, as a function of $\min (p(X), p(Y))$ or $\max (p(X), p(Y))$, respectively. The maximum value of $p_{\max }(X \wedge Y)$ obtained when varying Equation (2) over $p(Y)$ is equal to $p(X)$, so the upper bound in Fig. $2 \mathrm{~b}$ is simply the diagonal. All points lie inside the allowed regions, further adding to our confidence in a quantum explanation for the data.

\section{Co-occurrence of the effects}

So far we have seen that both order effects and conjunction fallacies are present in the data. However, quantum theory predicts that these effects should co-occur not just for the same stimuli but also in the same individuals. To

Table 2 Analysis of effects for the Bayesian repeated-measures ANOVA of the order effect constraint, with candidate type and the two sides of the constraint as the repeated measures

\begin{tabular}{ll}
\hline Effects & $\mathrm{BF}_{\text {Inclusion }}$ \\
\hline Side & $1.6 \times 10^{-2}$ \\
Candidate Type & $1.8 \times 10^{4}$ \\
Candidate Pair & $3.2 \times 10^{-5}$ \\
Side * Candidate Type & $4.0 \times 10^{-3}$ \\
Side * Candidate Pair & $5.3 \times 10^{-9}$ \\
Candidate Type * Candidate Pair & $2.7 \times 10^{-7}$ \\
Side * Candidate Type * Candidate Pair & $3.0 \times 10^{-13}$ \\
\hline
\end{tabular}

b

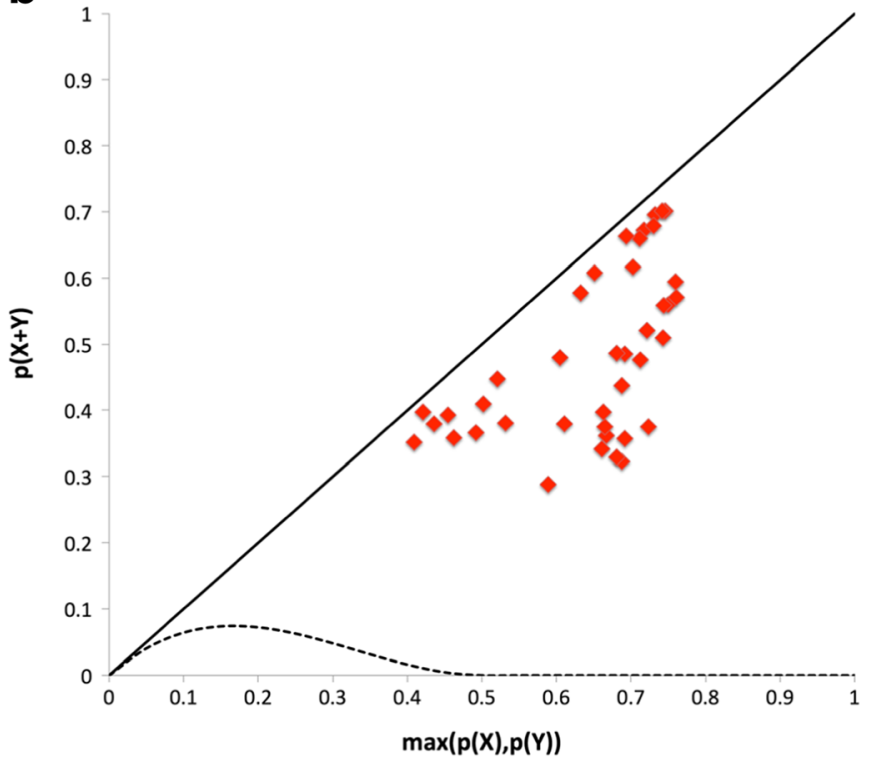

Conjunctions plotted against the maximum conjunct, all points lie below the diagonal, indicating the absence of any double conjunction fallacies. The dotted and solid lines represent the quantum bounds

demonstrate this, it is useful to have a single measure of the size of order effects and conjunction fallacies committed by a given participant for both the Democratic and Republican candidates. For each participant and choice of candidate, we can compute an order effect score and a conjunction fallacy score as follows. Let $N$ denote the event that a given candidate wins the nomination, and $A$ and $\bar{A}$ the events that a candidate wins or loses the primary A, respectively. The order effect score we computed is given by

$O E=\frac{1}{\sigma}\{|p(N \mid \bar{A}, B)-p(N \mid B, \bar{A})|+|p(N \mid \bar{B}, A)-p(N \mid A, \bar{B})|\}$

for the relevant primaries $A$ and $B$. This gives an indication of the size of order effects exhibited by each participant. Here, $\sigma$ is the standard deviation of the participant's responses for all questions about this candidate. Dividing by the standard deviation helps to reduce any effects due to participants not using the entire response scale.

The conjunctions we tested involved one primary and the party nomination (e.g., Trump wins TX and loses the nomination). The conjunction fallacy score we computed is therefore made up of two terms,

$$
C F=\frac{1}{\sigma} \sum_{\substack{X \in\{A, \bar{A}, B, \bar{B}\} \\ Y \in\{N, \bar{N}\}}}[\max \{p(X \wedge Y)-p(X), 0\}+\max \{p(X \wedge Y)-p(Y), 0\}] .
$$




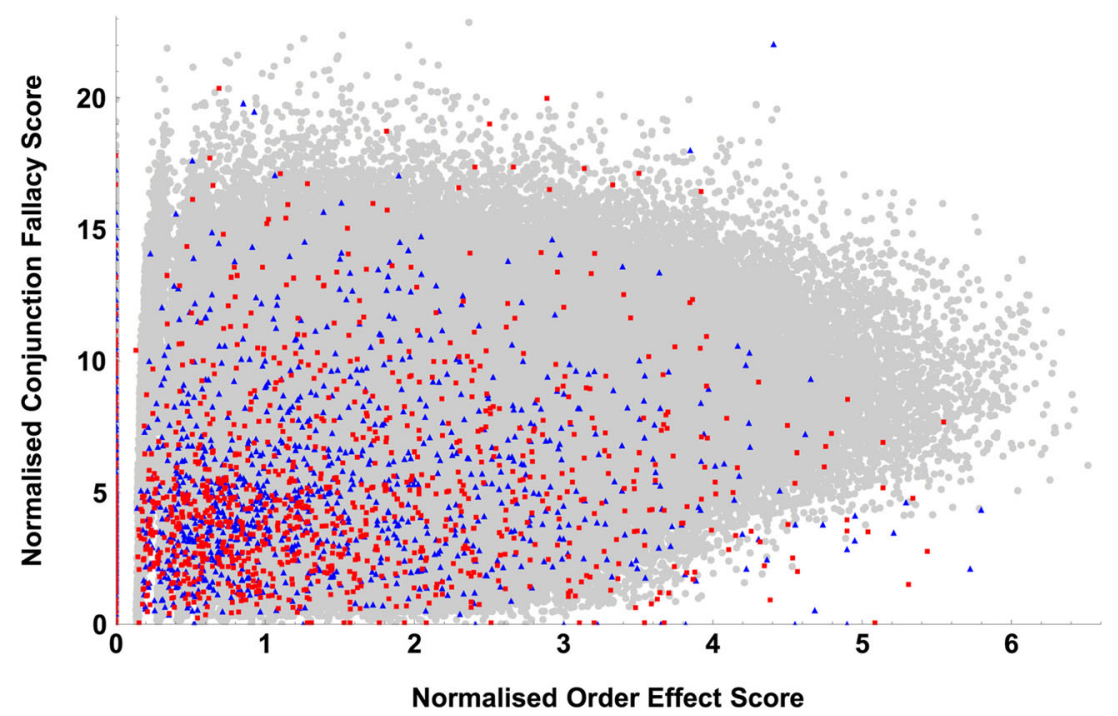

Fig. 3 Scatter plot of the normalized order effect and conjunction fallacy scores. The red square points represent data for Republican candidates and the blue triangle points represent data for Democrat candidates. Each

The first term compares the conjunction to one of the constituent events and the second term compares it to the other constituent event. This measure is sensitive both to the number of conjunction fallacies committed and to their size. Again, $\sigma$ is the standard deviation of the participant's responses for all question about this candidate.

Figure 3 plots the two scores, split up into responses for Democratic (blue points) and Republican candidates (red points). We can clearly see that the majority of participants display both order effects and conjunction fallacies. We can get additional evidence for the quantum model by examining how it predicts the co-occurrence of conjunction fallacies and order effects. To do this, we simulated a simple quantum model, exhausting the possible parameter space to get an impression of the degree of co-occurrence typical in such a model. Full details of the simulations are given in the supplementary materials. Results of the simulation are also plotted in Fig. 3 (gray points), and we can see that the data for the most part lie within the same region as the majority of the simulation results. Together these features provide additional evidence for a quantum model of these decisions.

\section{Individual differences}

To check for the effects of CRT and political ideology, we ran two Bayesian repeated-measures ANOVAs with either order effect score, Equation (3), or conjunction fallacy score, Equation (4), as the repeated measures, with CRT and ideology as between-subjects factors. The CRT and 10-pt ideology scales both had acceptable reliability $(\alpha=.77$ and $\alpha=.80$, point represents one participant. The gray points are the results of model simulations. (Color figure online)

respectively ${ }^{2}$ ), but to reduce noise and help even out numbers in each group, we collapsed the 10-point ideology scale down to a three category ideology score, with categories given by Liberal (0-3), Moderate (4-6), and Conservative (7-10). In this section, we also collapse results across candidates from the same party.

For the order effect score, a model with Candidate Type (Republican or Democrat) and CRT factors was preferred to all other models $\left(\mathrm{BF}_{\mathrm{M}}=18.4\right.$, strong evidence $)$ and to the null model $\left(\mathrm{BF}_{10}=185.6\right.$, extreme evidence $)$. The analysis of effects is shown in Table 3, and the full analysis is given in the Supplementary Material. It shows that the effect CRT has $\mathrm{BF}_{\text {inclusion }}=9.9$ (moderate evidence).

For the conjunction fallacy score, a model with CRT and Ideology factors was preferred to all other models $\left(\mathrm{BF}_{\mathrm{M}}=\right.$ 27.8 , strong evidence) and to the null model $\left(\mathrm{BF}_{10}=2.6 \times\right.$ $10^{4}$, extreme evidence). The analysis of effects is shown in Table 4, the full analysis is given in the Supplementary Material. It shows that the effect $\mathrm{CRT}$ has $\mathrm{BF}_{\text {inclusion }}=$ 304 (extreme evidence) and Ideology has $\mathrm{BF}_{\text {inclusion }}=3.1$ (weak evidence).

The behavior of the order effect score and conjunction fallacy score with varying CRT and ideology score is shown in Fig. 4. Figure $4 \mathrm{a}$ and $\mathrm{b}$ show how the order effect score varies across subgroups with differing ideology and CRT scores. There is no discernible pattern when grouping by ideology, but a clear trend towards decreasing order effect scores with increasing CRT score, in line with previous work showing the CRT is associated with more

\footnotetext{
${ }^{2}$ These are the values of Cronbach's alpha computed from our data set in JASP.
} 
Table 3 Analysis of effects for the Bayesian repeated-measures ANOVA of order effect scores, with candidate type as the repeated measure

\begin{tabular}{ll}
\hline Effects & BFInclusion \\
\hline Candidate Ttype & 2.75 \\
CRT & 9.94 \\
Ideology & 0.23 \\
Candidate Type $\times *$ CRT & 0.03 \\
Candidate Type $\times *$ Ideology & 0.08 \\
CRT $\times *$ Ideology & 0.02 \\
Candidate Type $\times *$ CRT $\times *$ Ideology & $4.81 \times 10^{-7}$ \\
\hline
\end{tabular}

deliberative thinking. Figure $4 \mathrm{c}$ and $\mathrm{d}$ show how the conjunction fallacy score varies across subgroups with differing ideology and CRT scores. There is a clear trend towards decreasing conjunction fallacy scores with increasing CRT scores, as expected if those with higher CRT score are more deliberative. For the Democrat candidates, the conjunction fallacy score is lowest for those identifying as liberal, while for Republican candidates the scores are lower for those identifying as conservative. Scores for both candidates are higher for those whose political ideology is moderate. One interpretation is that those with strong political beliefs may be more deliberative when answering these questions, especially when it comes to their preferred candidate.

We note briefly that our sample of mTurk participants is unlikely to be representative of the U.S. population as a whole. The proportion of participants classified as Liberal, Moderate, and Conservative was $54.0 \%, 28.2 \%$ and $17.8 \%$ respectively. Likewise, the proportion of participants scoring $0,1,2$, or 3 in the CRT was $15.8 \%, 22.1 \%, 25.3 \%$, and $36.8 \%$, respectively, the reverse of the original study of Frederick (2005), where the majority of participants had low scores. This may affect the generalizability of the individual differences analysis (Simons, Shoda, \& Lindsay, 2017), and it would be interesting

Table 4 Analysis of effects for the Bayesian repeated-measures ANOVA of conjunction fallacy scores, with candidate type as the repeated measure

\begin{tabular}{lc}
\hline Effects & $\mathrm{BF}_{\text {Inclusion }}$ \\
\hline Candidate type & 0.16 \\
CRT & 304.30 \\
Ideology & 3.12 \\
Candidate Type $\times$ CRT & 0.05 \\
Candidate Type $\times$ Ideology & 0.24 \\
CRT $\times$ Ideology & 0.01 \\
Candidate Type $\times$ CRT $\times$ Ideology & $2.81 \times 10^{-5}$ \\
\hline
\end{tabular}

in particular to examine how the results here replicate in a more ideologically representative sample.

\section{Alternative models}

The key advantage of quantum models of nonnormative behavior is that they unite what have often been thought of as disparate phenomena. As far as we are aware, no other models explain both order effects and conjunction fallacies as arising from the same underlying processes. Thus, a direct model comparison is difficult. However, we can take each effect separately and ask whether alternative models can account for the patterns of behavior observed.

A popular model of order effects is the belief adjustment model due to Hogarth and Einhorn (1992). In this model belief in a proposition is updated on receiving new evidence in a way that depends on the strength of the evidence relative to some reference point. The key challenge for any alternative model of order effects is to reproduce the order effect constraint in Equation (1). In the Supplementary Material, we prove this constraint is not obeyed by either common version of the belief adjustment model except under special conditions - essentially, that a candidate winning or losing a primary has an equal and opposite effect on the belief about the nomination. Such conditions are unlikely in the present study (e.g., if a candidate is judged a priori unlikely to win a particular primary such a loss will likely have little effect on belief about the eventual nomination; however, an unexpected win would likely greatly increase belief in the probability of winning the nomination).

There are a number of alternative heuristic models that can account for conjunction fallacies, but a recent quantitative model of interest is the probability theory plus noise model of Costello and Watts (2014). In this model, noise in the process of retrieving exemplars means that the expected value of a person's probability judgment $p_{E}(X)$ is squeezed toward one half, with a larger degree of noise and thus squeezing for conjunctions compared to single events, because these are more complex. The overall effect is that the expected value for the probability of a conjunction is squeezed such that it may become greater than one or both of the constituent events, giving a conjunction fallacy.

A key observation is that conjunction fallacies are only possible in this model if at least one of the constituent events has probability $<1 / 2$. With some algebra, given in full in the supplementary materials, we can show,

$p_{E}(X \wedge Y) \leq \min \left(p_{E}(X), p_{E}(Y)\right)$, if $\min \left(p_{E}(X), p_{E}(Y)\right)>\frac{1}{2}$,

a constraint not satisfied in the data (see Fig. 2a). We conclude that the probability plus noise model is unable to account for the data presented here. 

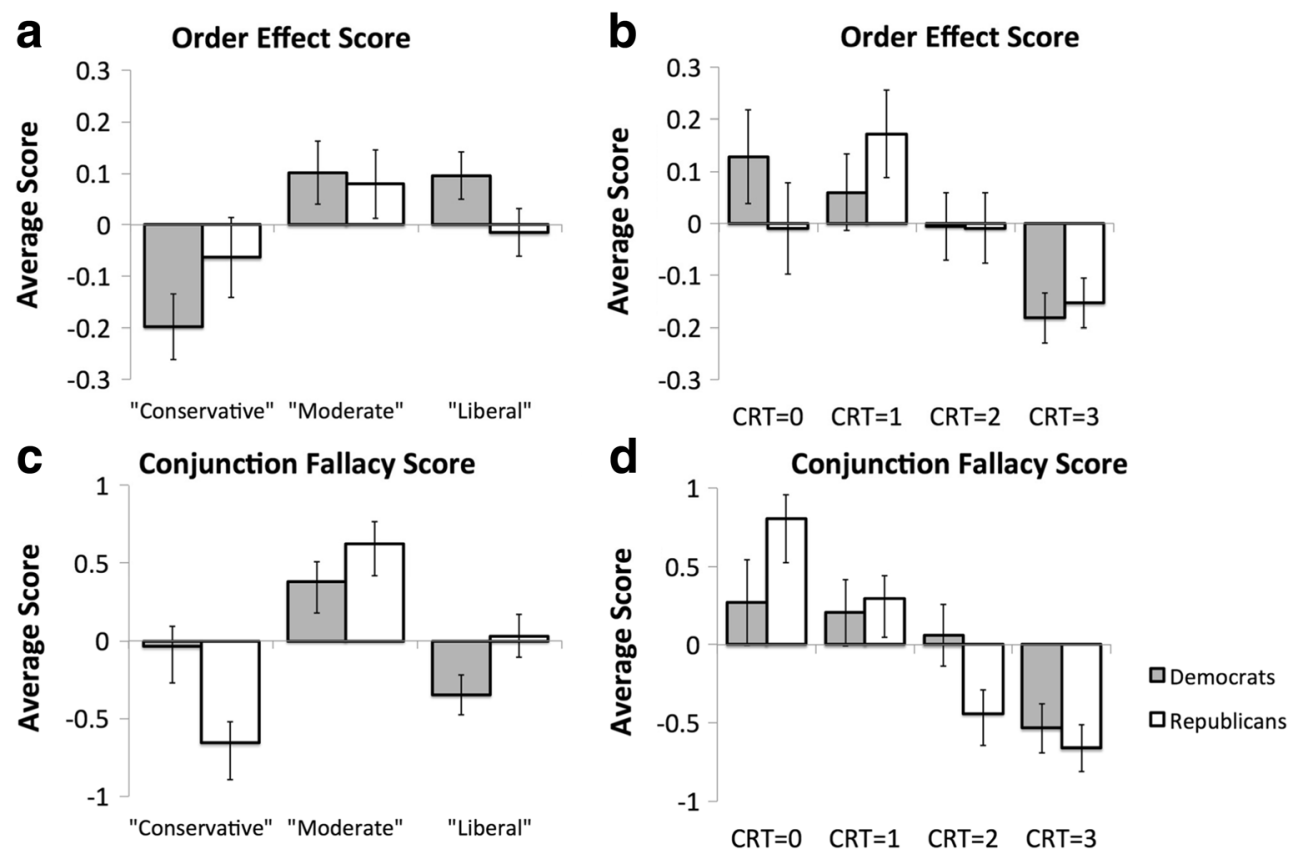

Fig. 4 Plots of the order effect and conjunction fallacy scores. These are plotted as the difference from the mean taken across CRT or ideology categories, removing any main effect of candidate type. Error bars show $S E$ of the mean

Thus, it is unclear that alternative models can account for the patterns of data observed even when considering the two effects separately.

\section{Conclusion}

The value of quantum models lies in their unification of disparate nonnormative behaviors. In this article, we tested two a priori and parameter free predictions of the quantum model, one for order effects and one for conjunction fallacies. The data are in good agreement with both, as well as validating the reasonable but previously untested prediction that these effects should co-occur. This gives us confidence in a quantum theoretic explanation for these violations of normative decision making. The results from the analysis of individual differences show performance on the CRT is a good predictor of deviations from normative behavior. In addition, ideology score is a good predictor of the size and frequency of conjunction fallacies, although not of order effects. Overall, these results add to a growing body of evidence that quantum models of judgment and decision-making are valuable tools for modeling human behavior.

The theoretical appropriateness of the quantum analysis is predicated on the idea that the events considered, winning the individual primaries and winning the relevant nomination, are incompatible in the minds of participants and must be considered sequentially. Incompatibility cannot be assessed directly and instead must be inferred from the presence of quantumlike behavioral effects (in particular, order effects). The key contribution of the present study is that we have taken a first step beyond this approach - the presence of one effect can be used to infer incompatibility, which can then be used to predict the presence of another effect. While incompatibility remains an empirical question, we hope this approach leads to improved predictive power for the quantum approach.

Acknowledgements J.M.Y. and J.S.T. were supported by NSF Grant SES-1326275.

\section{References}

Busemeyer, J., \& Bruza, P. (2012). Quantum models of cognition and decision. Cambridge: Cambridge University Press.

Busemeyer, J., Pothos, E., Franco, R., \& Trueblood, J. (2011). A quantum theoretical explanation for probability judgment errors. Psychological Review, 118, 193-218.

Costello, F., \& Watts, P. (2014). Surprisingly rational: Probability theory plus noise explains biases in judgment. Psychological Review, 121(3), 463-480.

Finucane, M. L., \& Gullion, C. M. (2010). Developing a tool for measuring the decision-making competence of older adults. Psychology and Aging, 25(2), 271-288.

Frederick, S. (2005). Cognitive reflection and decision making. Journal of Economic Perspectives, 19, 25-42.

Griffiths, T. L., Chater, N., Kemp, C., Perfors, A., \& Tenenbaum, J. B. (2010). Probabilistic models of cognition: Exploring representations and inductive biases. Trends in Cognitive Sciences, 14(8), 357-364.

Hogarth, R., \& Einhorn, H. (1992). Order effects in belief updating: The belief-adjustment model. Cognitive Psychology, 24, 1-55.

Kahneman, D., \& Tversky, A. (1972). On prediction and judgment. ORI Research Monographs, 12.

Pew Research Center. (2014). The ideological consistency scale. Retrieved from http://www.people-press.org/2014/06/12/appendixa-the-ideological-consistency-scale/ 
Simons, D. J., Shoda, Y., \& Lindsay, D. S. (2017). Constraints on Generality (COG): A proposed addition to all empirical papers. Retrieved from https://psyarxiv.com/w9e3r/

JASP Team. (2016). JASP [Computer software]. Retrieved from https:// jasp-stats.org

Toplak, M., West, R., \& Stanovich, K. (2011). The cognitive reflection test as a predictor of performance on heuristics-and-biases tasks. Memory \& Cognition, 39(7), 1275-1289.

Trueblood, J., \& Busemeyer, J. (2011). A quantum probability account of order effects in inference. Cognitive Science, 35, 1518-1552.

Tversky, A., \& Kahneman, D. (1975). Judgment under uncertainty: Heuristics and biases. In D. Wendt \& C. Vlek (Eds.), Utility, probability, and human decision making (pp. 141-162). New York: Springer.

Tversky, A., \& Kahneman, D. (1983). Extensional versus intuitive reasoning: The conjunction fallacy in probability judgment. Psychological Review, 90, 293-315.

Wang, Z., \& Busemeyer, J. (2013). A quantum question order model supported by empirical tests of an a priori and precise prediction. Topics in Cognitive Science, 5, 689-710.

Windschitl, P. D., \& Wells, G. L. (1996). Measuring psychological uncertainty: Verbal versus numeric methods. Journal of Experimental Psychology: Applied, 2(4), 343-364. 